\title{
Systemic oxidoreductive balance and vascular function in individuals without clinical manifestation of atherosclerosis
}

\author{
Marcin Majer ${ }^{1}$, Daniel Gackowski², Rafał Różalski², Agnieszka Siomek-Górecka², Ryszard Oliński², \\ Jacek Budzyński $i^{1,3}$
}

\begin{abstract}
${ }^{1}$ Clinic of Vascular and Internal Diseases, Jan Biziel University Hospital No. 2, Bydgoszcz, Poland

${ }^{2}$ Department of Clinical Biochemistry, Faculty of Pharmacy, Ludwik Rydygier Collegium Medicum in Bydgoszcz, Nicolaus Copernicus University in Torun, Poland

${ }^{3}$ Department of Vascular and Internal Diseases, Faculty of Health Sciences, Ludwik

Rydygier Collegium Medicum in Bydgoszcz, Nicolaus Copernicus University in Torun, Poland
\end{abstract}

Submitted: 9 July 2017

Accepted: 31 July 2017

Arch Med Sci Atheroscler Dis 2017; 2: e37-e45

DOI: https://doi.org/10.5114/amsad.2017.70501

Copyright @ 2017 Termedia \& Banach

\begin{abstract}
Introduction: Endothelial dysfunction is recognized as the earliest disorder in the development of atherosclerosis, in the pathogenesis of which oxidative stress plays a crucial role. The aim of this study was to determine the relationships between non-invasive parameters of vascular dysfunction and oxidative stress.

Material and methods: Forty-eight individuals without clinical manifestation of atherosclerosis were studied. The plasma concentrations of the following were determined in all 48 subjects: retinol, ascorbic acid, $\alpha$-tocopherol and uric acid, as well as the products of oxidative DNA damage repair: 8-oxo-7,8-dihydro-2'-deoxyguanosine (8-oxodG) in blood leukocytes and urine, and 8-oxo-7,8-dihydroguanine (8-oxoGua) in urine. The following parameters of vascular dysfunction were also examined: flow- (FMD) and nitroglycerin- (NMD) mediated dilatation of the brachial artery, pulse pressure $(\mathrm{PP})$, distensibility coefficient (DC), pulsation (PI) and resistance (RI) index, carotid intima-media thickness (cIMT), and ankle-brachial index (ABI).

Results: Individuals with an FMD value of $\geq 8.8 \%$ had significantly higher blood concentrations of antioxidative vitamins and lower concentrations of 8-oxodG in their urine and blood leukocytes than their counterparts. Blood concentration of alpha-tocopherol or ascorbic acid positively correlated with FMD, PI, RI, DC and ABI and negatively with PP and CIMT. The reverse was the case for 8-oxodG in urine and leukocytes. In multiple regression analysis, markers of oxidative DNA damage positively determined the variance in PP and $\mathrm{ABI}$.

Conclusions: In persons without clinical manifestation of atherosclerosis, oxidative stress was an independent factor associated with vascular wall dysfunction, and a better predictor than smoking and blood concentrations of glucose, lipids and creatinine.
\end{abstract}

Key words: oxidative stress, DNA oxidative damage markers, vascular function, flow-mediated dilatation, intima-media thickness, ankle-brachial index.

\author{
Corresponding author: \\ Dr Jacek Budzyński \\ Department of Vascular \\ and Internal Diseases \\ Jan Biziel University \\ Hospital No. 2 \\ 75 Ujejskiego St \\ 85-168 Bydgoszcz, Poland \\ Phone/fax: +48 523655148 \\ E-mail: budz@cps.pl
}




\section{Introduction}

Endothelial dysfunction plays a crucial role in atherosclerosis development and progression. It is an endpoint effect of all known atherosclerosis risk factors (e.g. smoking, hypertension, diabetes and hyperlipidemia) and promotes atherogenesis through its influence on vasodilation mechanisms, the stimulation of arterial thrombosis, and the migration and proliferation of vascular wall cells $[1,2]$. The most frequent cause of endothelial dysfunction is oxidative stress, which is an effect of the activation of redox signaling pathways through the influence of proatherogenic conditions, including high levels of low-density lipoprotein (LDL) and its oxidized form (ox-LDL) [3].

Endothelial dysfunction is recognized as a biomarker of early and subclinical injury of the vascular wall and assists in the cardiovascular risk stratification of patients with various comorbidities [4-7]. Finally, endothelial dysfunction leads to functional or morphological abnormality of the vascular wall, signs of which justify an increase in the intensity of the control of atherosclerosis risk factors. The most frequently used markers in the diagnosis of arterial wall deterioration, vessel performance and/or its hemodynamic consequences are: (a) increase in carotid intima-media thickness (cIMT), (b) decrease in ankle-brachial index (ABI); (c) brachial artery flow-mediated dilatation (FMD), which reflects vasorelaxation dependent on endothelial function, and endogenous nitric oxide release; this test is sometimes complemented by sublingual nitroglycerine application (nitroglycerine-mediated dilatation - NMD), which assesses the possibility of vasodilatation independent of endothelial function (endothelium-independent vasomotion), and makes it possible to evaluate the entire vasomotor reserve of the smooth muscles that form the vessel wall [8-13]; (d) tonometry of peripheral arteries (digital pulse amplitude tonometry or PAT) to calculate the passive hyperemia index, augmentation index and reactive hyperemia in response to ischemia (RH-PAT) [4, 11-13]; (e) arterial stiffness examination based on pulse pressure (PP) measurement (the difference between systolic and diastolic blood pressure, whereby an amplitude greater than $55 \mathrm{~mm}$ $\mathrm{Hg}$ shows increased cardiovascular risk), pulse wave shape and propagation (pulse wave velocity - PWV) registered in the proximal and distal points of the circulatory system; (f) arterial distensibility coefficient (DC), which is expressed as the difference between systolic and diastolic diameters of the common carotid artery divided by the product of values for the diastolic artery diameter and pulse pressure; (g) pulsatility and resistance indexes, increased values of which represent higher risk for atherosclerosis [4-13].
Although oxidative stress is directly involved in atherosclerosis development and progression, only a few studies on the relationships between circulating parameters of oxidative stress and vascular wall function are available. We hypothesized that a decreased level of antioxidant in blood and increased concentration of markers of oxidative tissue damage might help to identify individuals at risk of early atherosclerosis development, who seem to be most likely to benefit from therapeutic intervention. Therefore, we performed an investigation with the aim of assessing the relationships between selected parameters of vascular wall function and markers of systemic oxidoreductive balance in persons without clinical manifestation of atherosclerosis.

\section{Material and methods}

We studied 48 individuals without a history of atherosclerosis manifestation. The inclusion criteria were as follows: no history of clinical atherosclerosis manifestation, in the following forms: acute coronary syndrome, myocardial infarction, stroke, transient ischemic attack, intermittent claudication, aortic aneurysm or other vascular diseases; and aged over 18 and having the ability to give informed consent for participation in the study. The exclusion criteria were as follows: symptoms or history of any vascular diseases; hypertension, diabetes mellitus (as an equivalent of vascular diseases); chronic pharmacotherapy, intake of diet supplements which could affect oxidoreductive balance; the inability to perform a treadmill stress test; and history or physical signs of inflammatory diseases or neoplasm.

The following were undertaken for all the subjects who qualified for the study: medical history (symptoms, past history of diseases, use of stimulants, daily intake of vegetables and fruit, and use of diet supplements and vitamins); and physical examination and basic laboratory investigations (blood morphology, determinations of creatinine, sodium, potassium, glucose, alanine aminotransferase, C-reactive protein, total, LDL, and HDL cholesterols, and triglycerides).

\section{Parameters of oxidoreductive balance}

Detailed examinations of oxidoreductive balance were performed, as previously described [14-16]:

- in plasma: the concentration of high-sensitivity C-reactive protein (hs-CRP), as well as retinol (vitamin A), ascorbic acid (vitamin C), tocopherol (vitamin E), and uric acid;

- in blood leukocyte DNA: 8-oxo-7,8-dihydro2'-deoxyguanosine (8-oxodG);

- urinary excretion of 8-oxo-7,8-dihydroguanine (8-oxoGua) and 8-oxo-7,8-dihydro-2'-deoxygua nosine (8-oxodG); 
- antioxidative status (AS) - defined as the geometric mean concentration of antioxidant vitamins determined;

- prooxidative status (PS) - defined as the geometric mean concentration of DNA damage products determined;

- redox index: defined as the ratio of PS to AS.

\section{Parameters of vascular dysfunction}

The following parameters of vascular wall function were calculated [7-13]:

- brachial artery FMD, with the measurement of changes in internal brachial artery systolic diameter after 5 min of arm compression using a cuff at a pressure that is $30 \mathrm{~mm} \mathrm{Hg}$ greater than systolic blood pressure; this was calculated according to the following formula: $\operatorname{FMD}(\%)=$ $100 \% \times$ (systolic diameter after arm ischemia systolic diameter before arm ischemia)/systolic diameter before arm ischemia; this parameter reflects endothelium-dependent vasodilatation;

- NMD of brachial artery one minute after sublingual (sl.) application of $0.4 \mathrm{mg}$ nitroglycerine (NTG) in spray form; this was calculated in accordance with the formula: $\mathrm{NMD}(\%)=100 \%$ $\times$ (systolic diameter after sl. NTG application - systolic diameter before sl. NTG application)/ systolic diameter before sl. NTG application; this parameter reflects endothelium-independent vasodilatation, which is mainly related to the relaxation of vascular smooth muscle;

- PP, calculated as the difference between systolic and diastolic blood pressures measured for the left brachial artery;

- pulsation index (PI), calculated using parameters obtained during Doppler ultrasound examination of the brachial artery in accordance with the formula: $\mathrm{PI}=$ (peak systolic velocity (PSV) - minimum diastolic blood velocity)/ mean blood flow velocity;

- resistance index (RI), calculated using parameters obtained during Doppler ultrasound examination of the brachial artery in accordance with the formula: $\mathrm{RI}=$ (PSV - peak diastolic blood velocity)/PSV;

- cIMT, measured in the common carotid artery $2 \mathrm{~cm}$ below the bifurcation and presented as the mean of the two values from both sides;

- common carotid artery internal diastolic (CCAdd) and systolic (CCAsd) diameters, presented as the mean of the two values from both sides;

- relative common carotid artery wall thickness (cWT), calculated in accordance with the formula: $\mathrm{CWT}=2 \times \mathrm{cIMT/CCAdd}$, presented as the mean of two values from both sides;

- the difference between systolic and diastolic diameters of the common carotid artery, calcu- lated as DD = CCAsd - CCAdd, presented as the mean of two values from both sides;

- DC, which was calculated in accordance with the formula: $D C=2(\mathrm{DD} / \mathrm{CCAdd}) / \mathrm{PP}$, where PP was expressed in $10^{3} / \mathrm{kPa}$;

- elasticity modulus (EM), which was calculated in accordance with the formula: $E M=P P$ $\times$ CCAsd/CCAdd;

- $A B I$, which was calculated as a quotient of the systolic blood pressures measured at the ankle and in the upper arm, presented as the lower of two values from both sides.

\section{Bioethics}

The study protocol was approved by the local Bioethical Committee, and the study was conducted in compliance with the Declaration of Helsinki for medical research. All study participants signed informed consent for participation in the study.

\section{Statistical analysis}

Statistical analysis was conducted using licensed versions of statistical software Statistica (a data analysis software system), StatSoft, Inc. (2015), version 12. The normal distribution of the study variables was checked using the Shapiro-Wilk test. The results were mainly presented as the mean \pm standard deviation or $n, \%$. The statistical significance of the differences between groups was verified using Student's $t$-test and the $\chi^{2}$ test. The statistical significance level was set at a $p$-value $<0.05$. Pearson's correlations were determined. Stepwise backward multiple regression (using the General Regression Models module of Statistica) was applied to evaluate the relationships between the values of the respective parameters of vascular wall function and the parameters of oxidoreductive balance. Subjects' age, gender, and the values of the above-mentioned parameters of oxidoreductive balance and laboratory tests were selected as the independent variables, and the above-listed parameters of vascular wall function were used as the dependent variables.

\section{Results}

The participants of our study were divided into two groups in relation to the median FMD value (Table I). Subjects with better endothelial function (FMD $\geq 8.88 \%$ ) had higher plasma concentrations of ascorbic acid, retinol and $\alpha$-tocopherol, as well as significantly lower concentrations of 8-oxodG in their urine and leukocytes than their counterparts. Moreover, they were younger, less prevalently male, had lower creatinine blood concentration, and had similar blood concentrations of plasma lipids and glucose. Greater endothelium-dependent vasodilatation was also related to 
Table I. Clinical and biochemical parameters in patients divided in relation to the median value of flow-mediated brachial artery dilatation (FMD)

\begin{tabular}{|c|c|c|c|}
\hline Parameter & $\begin{aligned} \mathrm{FMD} & \geq 8.88 \% \\
N & =24\end{aligned}$ & $\begin{aligned} \mathrm{FMD} & <8.88 \% \\
N & =24\end{aligned}$ & $P$-value \\
\hline Age [years] & $54.88 \pm 4.28$ & $58.04 \pm 4.76$ & 0.02 \\
\hline Male gender, $n(\%)$ & $10(42)$ & $18(75)$ & $<0.01$ \\
\hline BMI $\left[\mathrm{kg} / \mathrm{m}^{2}\right]$ & $27.28 \pm 4.14$ & $27.83 \pm 3.11$ & 0.61 \\
\hline Current smoker, $n(\%)$ & $5(21)$ & $3(12.5)$ & 0.51 \\
\hline Plasma hs-CRP [mg/l] & $2.02 \pm 1.68$ & $1.48 \pm 1.01$ & 0.14 \\
\hline Plasma ascorbic acid $[\mu \mathrm{M}]$ & $44.58 \pm 21.08$ & $32.57 \pm 18.08$ & 0.04 \\
\hline Plasma retinol $[\mu \mathrm{M}]$ & $2.93 \pm 0.45$ & $2.53 \pm 0.76$ & 0.03 \\
\hline Plasma $\alpha$-tocopherol $[\mu \mathrm{M}]$ & $35.96 \pm 6.67$ & $31.83 \pm 5.51$ & 0.02 \\
\hline Plasma uric acid $[\mu \mathrm{M}]$ & $335.54 \pm 53.61$ & $356.69 \pm 69.30$ & 0.24 \\
\hline 8-oxoGua in urine $[\mathrm{nmol} / \mathrm{mmol}$ creatinine $]$ & $7.05 \pm 2.84$ & $10.26 \pm 7.73$ & 0.062 \\
\hline 8-oxodG in urine $[\mathrm{nmol} / \mathrm{mmol}$ creatinine] & $2.18 \pm 0.83$ & $2.82 \pm 1.12$ & 0.027 \\
\hline 8-oxodG in leukocytes (8-oxodG $/ 10^{6} \mathrm{dG}$ ) & $5.97 \pm 1.49$ & $7.29 \pm 2.38$ & 0.03 \\
\hline Creatinine $[\mathrm{mg} / \mathrm{dl}]$ & $1.01 \pm 0.13$ & $1.12 \pm 0.16$ & 0.01 \\
\hline Blood glucose concentration [mg/dl] & $98.64 \pm 18.69$ & $94.52 \pm 17.58$ & 0.44 \\
\hline Total cholesterol [mg/dl] & $222.28 \pm 44.43$ & $220.00 \pm 29.88$ & 0.84 \\
\hline HDL cholesterol [mg/dl] & $59.76 \pm 15.20$ & $58.22 \pm 9.72$ & 0.68 \\
\hline LDL cholesterol [mg/dl] & $138.60 \pm 34.27$ & $135.00 \pm 26.44$ & 0.69 \\
\hline Triglycerides [mg/dl] & $118.48 \pm 36.53$ & $118.43 \pm 47.78$ & 0.99 \\
\hline FMD & $9.28 \pm 5.55$ & $3.52 \pm 2.99$ & $<0.001$ \\
\hline NMD & $10.61 \pm 2.99$ & $9.98 \pm 4.19$ & 0.51 \\
\hline Pulse pressure [mm Hg] & $43.60 \pm 7.97$ & $48.13 \pm 9.73$ & 0.084 \\
\hline $\begin{array}{l}\text { Mean difference between systolic and } \\
\text { diastolic internal diameter of carotid arteries }\end{array}$ & $0.39 \pm 0.21$ & $0.40 \pm 0.17$ & 0.89 \\
\hline Carotid wall thickness (cWT) & $0.23 \pm 0.04$ & $0.25 \pm 0.06$ & 0.12 \\
\hline Pulsatility index (PI) & $1.65 \pm 0.081$ & $1.73 \pm 0.10$ & $<0.01$ \\
\hline Resistance index (RI) & $0.79 \pm 0.06$ & $0.84 \pm 0.07$ & $<0.01$ \\
\hline Distensibility coefficient (DC) & $20.85 \pm 11.64$ & $15.80 \pm 6.73$ & 0.08 \\
\hline Elasticity modulus (EM) & $51.20 \pm 9.42$ & $41.25 \pm 8.03$ & $<0.001$ \\
\hline Mean cIMT $[\mathrm{mm}]$ & $0.76 \pm 0.14$ & $0.86 \pm 0.18$ & 0.04 \\
\hline Mean ABI & $1.21 \pm 0.17$ & $1.21 \pm 0.19$ & 0.98 \\
\hline
\end{tabular}

Data presented as mean \pm standard deviation (SD) and number $(n, \%)$ of qualitative variables. The statistical significance level was set at a p-value $<0.05 ; 8$-oxoGua in urine - concentration of 8-oxoguanine in urine calculated in relation to the concentration of creatinine, 8-oxodG in urine - concentration of 8-oxo-7,8-dihydro-2'-deoxyguanosine in urine calculated in relation to the concentration of creatinine, 8-oxodG in leukocytes - 8-oxo-7,8-dihydro-2'-deoxyguanosine content in leukocytes, BMI-body mass index, hs-CRP-plasma concentration of $C$-reactive protein determined using the high-sensitivity method, HDL - high-density lipoprotein, LDL - low-density lipoprotein, FMD - flow-mediated dilatation of brachial artery, NMD - nitroglycerin-mediated dilatation of brachial artery, cWT-relative carotid wall thickness, cIMT - carotid intima-media thickness, $A B I$ - ankle-brachial index. 
greater values of EM, and lower PI, RI, PP (not significant) and CIMT (Table I). Similar split analyses in relation to the median value were performed for NMD, PP, PI, RI, CWT, DC, EM, CIMT and ABI. However, the analyses did not show statistically significant differences in the oxidoreductive balance parameters between the groups of subjects with respect to the aforementioned parameters (data not presented in detail).

In univariate analysis, the plasma concentrations of antioxidative substances, such as ascorbic acid, $\alpha$-tocopherol, AS and PS, were positively correlated with parameters of arterial wall function (e.g. FMD) or hemodynamics in leg arteries (ABI), and negatively with indices of arterial stiffness or narrowing (e.g. PI, RI and cIMT) (Table II). On the other hand, an inverse pattern of relationships was found between the above-mentioned parameters (e.g. PP, PI, RI, DC, EM, ABI) and the oxidative stress indices (e.g. DNA damage markers). To determine which of the factors that were significant in univariate analysis were independent factors that affected vascular wall function, we performed stepwise backward multiple regression analysis (Table III). We found that the FMD value was independently determined only by the participants' age and gender, and that none of the factors analyzed appeared to be an independent predictor of DC or EM values. However, the other parameters of vascular wall function were independently related to parameters of oxidoreductive balance. PP independently correlated with 8-oxodG concentration in leukocytes; PI and RI were significantly related to ascorbic acid blood concentration; and CIMT and $A B I$ values were independently related to AS and PS, respectively (Table III). However, the adjusted determination coefficients of these multiple regression equations were not high (9-59\%).

\section{Discussion}

In our study, we examined the associations between biomarkers of antioxidative balance and parameters of vascular wall function in individuals without clinical manifestation of atherosclerosis. Subjects with better endothelial function, expressed by an FMD value $\geq 8.8 \%$, had higher plasma concentrations of vitamins $A, C$ and $E$ and lower concentrations of DNA injury products in urine and blood leukocytes compared to their counterparts (Table I). It should be underlined that, apart from being younger, being female, and having a lower blood creatinine concentration, none of the factors determined, including smoking status, blood glucose and plasma lipids, significantly discriminated individuals with respect to differences in endothelial function. Moreover, we also found some weak but statistically significant correlations between numerous parameters of vessel

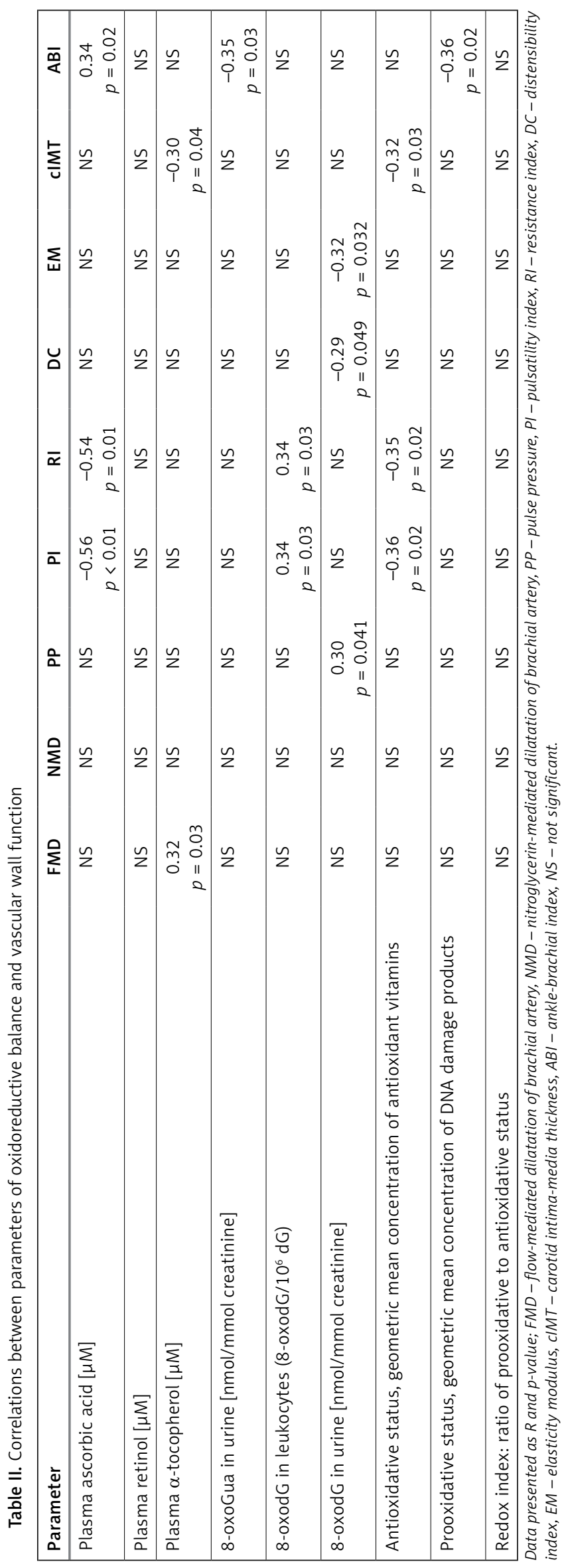


Table III. Stepwise backward multiple regression analysis for respective parameters determining vascular wall function

\begin{tabular}{|c|c|c|c|c|}
\hline $\begin{array}{l}\text { Dependent/ } \\
\text { independent variable }\end{array}$ & $\beta$ & Standard error for $\beta$ & $t(48)$ & $P$-value \\
\hline \multicolumn{5}{|c|}{ FMD: $R^{2}=0.30 ;$ adjusted $R^{2}=0.26 ; F=0.54 ; p=0.02$} \\
\hline Constant & & & -2.49 & 0.02 \\
\hline Age & -0.43 & 0.14 & -3.02 & $<0.001$ \\
\hline Female gender & 0.38 & 0.14 & 2.71 & 0.01 \\
\hline \multicolumn{5}{|c|}{ PP: $R^{2}=0.14 ;$ adjusted $R^{2}=0.11 ; F=0.57 ; p=0.02$} \\
\hline Constant & & & 12.39 & $<0.001$ \\
\hline $\begin{array}{l}\text { 8-oxodG in } \\
\text { leukocytes }\end{array}$ & 0.37 & 0.15 & 2.38 & 0.022 \\
\hline \multicolumn{5}{|c|}{$\mathrm{PI}: R^{2}=0.61 ;$ adjusted $R^{2}=0.59 ; F=27.49 ; p<0.001$} \\
\hline Constant & & & -3.88 & $<0.001$ \\
\hline Female gender & 0.52 & 0.11 & 4.58 & $<0.001$ \\
\hline Ascorbic acid & -0.33 & 0.11 & -2.90 & 0.01 \\
\hline \multicolumn{5}{|c|}{$\mathrm{RI}: R^{2}=0.59 ;$ adjusted $R^{2}=0.57 ; F=25.71 ; p<0.001$} \\
\hline Constant & & & -3.95 & $<0.001$ \\
\hline Female gender & 0.52 & 0.12 & 4.41 & $<0.001$ \\
\hline Ascorbic acid & -0.31 & 0.12 & -2.67 & 0.01 \\
\hline \multicolumn{5}{|c|}{ cIMT: $R^{2}=0.11 ;$ adjusted $R^{2}=0.09 ; F=5.9 ; p=0.02$} \\
\hline Constant & & & 9.88 & $<0.001$ \\
\hline AS & -0.34 & 0.14 & -2.43 & 0.02 \\
\hline \multicolumn{5}{|c|}{$\mathrm{ABI}: R^{2}=0.11 ;$ adjusted $R^{2}=0.09 ; F=4.65 ; p=0.04$} \\
\hline Constant & & & 28.01 & $<0.001$ \\
\hline PS & -0.33 & 0.15 & -2.16 & 0.04 \\
\hline
\end{tabular}

Data presented as $R$ and p-value; FMD - flow-mediated dilatation of brachial artery, PP - pulse pressure, PI - pulsatility index, $R I$ - resistance index, CIMT - carotid intima-media thickness, AS - antioxidative status defined as the geometric mean concentration of antioxidant vitamins, $A B I$ - ankle-brachial index, PS - prooxidative status defined as the geometric mean concentration of DNA damage products.

wall function and oxidative stress biomarkers (Table II), which confirmed the vasoprotective activity of antioxidants and the harmful effect of oxidative stress, markers of which, such as products of DNA damage, were related to worse vascular wall function. In multifactorial analysis, parameters of oxidoreductive balance independently determined the variances of PP, PI, RI, CIMT and $\mathrm{ABI}$, although the equations obtained had low determination coefficient values (Table III).

The results obtained by us corroborate previously published data. However, the majority of the previous research concerned the effects of dietary antioxidant intake or supplementation rather than blood concentration of antioxidative vitamins. In a study by Joris and Mensink [17], the supplementation of high doses of vitamin $E$ (tocopherol) increased FMD, whereas treatment with vitamin D did not appear to have this effect [17]. A study by Ives et al. [18] on patients with chronic obstructive lung disease found that a mixture of vitamin $C$, vitamin $E$, and $\alpha$-lipoic acid significantly improved FMD, and this effect was negatively correlated with baseline plasma vitamin E concentration. Improvement of endothelial function after antioxidant supplementation was also observed by Khan et al. [19] following consumption of blackcurrant juice with high levels of vitamin C content and polyphenols, by Johnson et al. [20] as a result of acute vitamin $C$ supplementation, and by Uzun et al. [21] after supplementation with vitamin $\mathrm{C}$ alone or its combination with vitamin E. A meta-analysis by Ashor et al. [22] revealed age-dependent improvement of endothelial function in investigations in which vitamin $\mathrm{C}$ (500-2000 mg/day) and vitamin E (300-1800 IU/ 
day) were supplemented alone, but not in studies in which these substances were co-administered. However, Chen et al. [23] did not observe improvement in FMD, PAT or PWV in 45 patients with CAD after 6 weeks of a diet containing almonds with high vitamin $\mathrm{E}$ content, in spite of a significant increase in blood concentration of $\alpha$-tocopherol. Similarly, in a study by Goldstein et al. [24], 4 weeks of treatment with high doses of vitamin C did not change the PAT parameters.

The relationships between vasodilatation reserve and blood or urine concentration of antioxidants and/or products of oxidative tissue injury have been studied in only a few investigations. Their results also corroborate our observations concerning both endothelial function (FMD) and immediate indices of arterial stiffness (PP, DC and EM). In a study by Hampson et al. [25], the balance of circulating $\alpha$ - and $\gamma$-tocopherol was negatively associated with arterial stiffness. Rimoldi et al. [26] found a significant correlation between changes in plasma ascorbic acid level and FMD after 4 weeks of oral supplementation with vitamins C (1 g/day) and E (400 IU/day) or a placebo. Moreover, in other studies, the blood concentrations of oxidative stress parameters were associated with the prevalence of major adverse cardiovascular events, which are recognized as the clinical endpoints of endothelial dysfunction. Nagao et al. [27] found that serum $\alpha$-tocopherol level in Japanese women was inversely associated with total stroke mortality, and a meta-analysis by Chen et al. [28] also suggested a significant inverse relationship between circulating vitamin $\mathrm{C}$ and risk of stroke. The association between circulating $\alpha$-tocopherol and risk of coronary artery disease (CAD) was also confirmed in a meta-analysis by Li et al. [29]. These data support our knowledge concerning cardio- and vasoprotective effects of antioxidants, which were also demonstrated in our study on the basis of the selected parameters of vascular wall function in individuals without clinical manifestation of atherosclerosis.

We feel that the outcomes of our multifactorial analysis require further commentary. We obtained only weak associations in multiple regression, in which age, gender and the values of the respective parameters of oxidoreductive balance explained only $9-59 \%$ of the variance of the vascular wall function parameters analyzed (Table III). It was also surprising that smoking, blood glucose and plasma lipids did not discriminate subjects with respect to differences in endothelial function (Table I). On the other hand, this observation suggests that parameters of oxidoreductive balance might be better predictors of early vascular injury than classical risk factors, but this requires further evaluation. These findings confirm atherosclerosis as a multifactorial disorder and suggest either the importance of other factors (e.g. infection, inflammation, genetic factors) leading to vessel injury or individually different susceptibility for alterations in vessel function in response to harmful factors, including pro- and antioxidative status. The latter explanation seems to be reasonable in the light of data indicating that endothelial dysfunction was diagnosed using available tests in only $46 \%$ of patients with stable angina pectoris, in $66 \%$ of patients with three atherosclerosis risk factors, but without significant narrowing of coronary arteries, and in $33 \%$ of patients with non-cardiac chest pain [30]. In our multifactorial analysis, we also found that early stages of vascular dysfunction (expressed by FMD, PI and $\mathrm{RI}$ ) were related to separate parameters of oxidoreductive balance (ascorbic acid, and 8-oxodG in leukocytes), but the more advanced stages of atherosclerosis which affected morphological (CIMT) and hemodynamic $(\mathrm{AB})$ indices of vascular injury were explained by a combination of parameters, such as AS and PS. This may suggest that, in the later stages of atherosclerosis, what is important for its progression may not be the effect of a single antioxidant, but rather the combined effect of a number of antioxidative substances (AS) which have to balance the PS. This may explain the failure of investigations to find a clear relationship between atherosclerosis progression and single antioxidant supplementation.

Unfortunately, as with most authors, we could not avoid some methodological shortcomings that could have influenced the strength of the deductions based on our results, in spite of obtaining statistically significant effects. Firstly, the number of subjects included in the study was relatively small. Secondly, the inclusion criteria were based on participants' medical history and their medical documentation, but not on invasive examinations such as coronarography or angiography of the peripheral arteries. On the other hand, carotid artery ultrasound with cIMT determination and $A B I$ are recognized as useful screening tools for the early diagnosis of vascular injury and the stratification of patients' risk [7]. Thirdly, the values of the parameters of oxidoreductive balance are dependent on many factors, which are sometimes difficult to identify in clinical examination but which may be potential sources of laboratory errors and mistakes in interpretation. In our study we excluded patients with neoplastic and inflammatory diseases and individuals taking synthetic vitamins, as well as patients with chronic diseases and elevated blood concentrations of hs-CRP and CRP.

In conclusion, in patients without clinical manifestation of atherosclerosis, oxidative stress, de- 
termined on the basis of parameters detected in plasma (antioxidative vitamins), blood leukocytes, and urine, was an independent factor associated with vascular wall dysfunction and a better predictor than smoking and blood concentrations of glucose, lipids and creatinine. Further studies are needed to evaluate the role of oxidative stress parameters as biomarkers of endothelial dysfunction, which is recognized as the earliest stage of atherosclerosis development.

\section{Conflict of interest}

The authors declare no conflict of interest.

\section{References}

1. Pellegrino T, Storto G, Filardi PP, et al. Relationship between brachial artery flow-mediated dilation and coronary flow reserve in patients with peripheral artery disease. J Nucl Med 2005; 46: 1997-2002.

2. Santos-García D, Blanco M, Serena J, Rodríguez-Yáñez M, Leira R, Castillo J. Impaired brachial flow-mediated dilation is a predictor of a new-onset vascular event after stroke. Cerebrovasc Dis 2011; 32: 155-62.

3. Li Y, Pagano PJ. Microvascular NADPH oxidase in health and disease. Free Radic Biol Med 2017; 109: 33-47.

4. Rubinshtein R, Kuvin JT, Soffler M, et al. Assessment of endothelial function by non-invasive peripheral arterial tonometry predicts late cardiovascular adverse events. Eur Heart J 2010; 31: 1142-8.

5. Al Rifai M, DeFillippis AP, McEvoy JW, et al. The relationship between smoking intensity and subclinical cardiovascular injury: the Multi-Ethnic Study of Atherosclerosis (MESA). Atherosclerosis 2017; 258: 119-30.

6. Frolow M, Drozdz A, Kowalewska A, Nizankowski R, Chlopicki S. Comprehensive assessment of vascular health in patients: towards endothelium-guided therapy. Pharmacol Rep 2015; 67: 786-92.

7. Yuan C, Wang J, Ying M. Predictive value of carotid distensibility coefficient for cardiovascular diseases and all-cause mortality: a meta-analysis. PLoS One 2016; 11: e0152799.

8. Husmann M, Dörffler-Melly J, Kalka C, Diehm N, Baumgartner I, Silvestro A. Successful lower extremity angioplasty improves brachial artery flow-mediated dilation in patients with peripheral arterial disease. J Vasc Surg 2008; 48: 1211-6.

9. Unal O, Karatepe O, Ugurlucan M, Koc B, Filizcan U, Aksoy $M$. Effects of lower extremity revascularization on the endothelial functions measured with noninvasive brachial artery flow-mediated dilatation. Ann Vasc Surg 2011; 25: 969-74.

10. Budzyński J, Wiśniewska J, Wasielewski M, Suppan K. The effect of superficial femoral artery stenting and some atherosclerosis risk factors on changes in selected global endothelial function tests in patients with chronic lower limb ischemia. A pilot study. Postep Kardiol Interw 2012; 8: 205-15.

11. TASC II Working Group. Patel S, Celermajer DS. Assessment of vascular disease using arterial flow mediated dilatation. Pharmacol Rep 2006; 58 Suppl: 3-7.

12. Corretti MC, Anderson TJ, Benjamin EJ, et al.; International Brachial Artery Reactivity Task Force. Guidelines for the ultrasound assessment of endothelial-dependent flow-mediated vasodilation of the brachial artery: a report of the International Brachial Artery Reactivity Task Force. J Am Coll Cardiol 2002; 39: 257-65.

13. Aizer J, Karlson EW, Chibnik LB, et al. A controlled com parison of brachial artery flow mediated dilation (FMD) and digital pulse amplitude tonometry (PAT) in the assessment of endothelial function in systemic lupus erythematosus. Lupus 2009; 18: 235-42.

14. Rozalski R, Migdalski A, Gackowski D, et al. Does morphology of carotid plaque depend on patient's oxidative stress? Clin Biochem 2013; 46: 1030-5.

15. Foksinski M, Gackowski D, Rozalski R, et al. Effects of basal level of antioxidants on oxidative DNA damage in humans. Eur J Nutr 2007; 46: 174-80.

16. Gackowski D, Rozalski R, Roszkowski K, Jawien A, Foksinski $M$, Olinski R. 8-Oxo-7,8-dihydroguanine and 8-oxo-7,8-dihydro-2'-deoxyguanosine levels in human urine do not depend on diet. Free Radic Res 2001; 35: 825-32.

17. Joris PJ, Mensink RP. Effects of supplementation with the fat-soluble vitamins $E$ and $D$ on fasting flow-mediated vasodilation in adults: a meta-analysis of randomized controlled trials. Nutrients 2015; 7: 1728-43.

18. Ives SJ, Harris RA, Witman MA, et al. Vascular dysfunction and chronic obstructive pulmonary disease: the role of redox balance. Hypertension 2014; 63: 459-67.

19. Khan F, Ray S, Craigie AM, et al. Lowering of oxidative stress improves endothelial function in healthy subjects with habitually low intake of fruit and vegetables: a randomized controlled trial of antioxidant- and polyphenol-rich blackcurrant juice. Free Radic Biol Med 2014; 72: 232-7.

20. Johnson BD, Mather KJ, Newcomer SC, Mickleborough TD, Wallace JP. Vitamin C prevents the acute decline of flow-mediated dilation after altered shear rate patterns. Appl Physiol Nutr Metab 2013; 38: 268-74.

21. Uzun A, Yener U, Cicek OF, et al. Does vitamin C or its combination with vitamin $\mathrm{E}$ improve radial artery endothelium-dependent vasodilatation in patients awaiting coronary artery bypass surgery? Cardiovasc J Afr 2013; 24: 255-9.

22. Ashor AW, Siervo M, Lara J, Oggioni C, Afshar S, Mathers JC. Effect of vitamin C and vitamin E supplementation on endothelial function: a systematic review and meta-analysis of randomised controlled trials. $\mathrm{Br} J \mathrm{Nutr}$ 2015; 113: 1182-94.

23. Chen CY, Holbrook M, Duess MA, et al. Effect of almond consumption on vascular function in patients with coronary artery disease: a randomized, controlled, crossover trial. Nutr J 2015; 14: 61.

24. Goldstein BH, Sandelin AM, Golbus JR, et al. Impact of vitamin $C$ on endothelial function and exercise capacity in patients with a Fontan circulation. Congenit Heart Dis 2012; 7: 226-34.

25. Hampson G, Edwards S, Sankaralingam A, et al. Circulating concentrations of vitamin $\mathrm{E}$ isomers: association with bone turnover and arterial stiffness in post-menopausal women. Bone 2015; 81: 407-12.

26. Rimoldi SF, Sartori C, Rexhaj E, et al. Antioxidants improve vascular function in children conceived by assisted reproductive technologies: a randomized double-blind placebo-controlled trial. Eur J Prev Cardiol 2015; 22: 1399-407.

27. Nagao M, Moriyama Y, Yamagishi K, Iso H, Tamakoshi A; JACC Study Group. Relation of serum alpha-and gamma-tocopherol levels to cardiovascular disease-related mortality among Japanese men and women. J Epidemiol 2012; 22: 402-10. 
28. Chen GC, Lu DB, Pang Z, Liu QF. Vitamin C intake, circulating vitamin $C$ and risk of stroke: a meta-analysis of prospective studies. J Am Heart Assoc 2013; 2: e000329.

29. Li G, Li Y, Chen X, Sun H, Hou X, Shi J. Circulating tocopherols and risk of coronary artery disease: a systematic review and meta-analysis. Eur J Prev Cardiol 2016; 23: 748-57.

30. Fichtlscherer S, Breuer S, Zeiher AM. Prognostic value of systematic endothelial dysfunction in patients with acute coronary syndromes: further evidence for the existence of the "vulnerable" patient. Circulation 2004; 110: 1926-32. 